\title{
A Rare Case of Periprosthetic Streptobacillosis - Rapid Identification via Nanopore Sequencing After Inconclusive VITEK MS Results
}

Riccardo Spott ( $\nabla$ riccardo.spott@med.uni-jena.de )

Institute for Infectious Diseases and Infection Control, Jena University Hospital

Benjamin Schleenvoigt

Institute for Infectious Diseases and Infection Control, Jena University Hospital

Birgit Edel

Institute for Medical Microbiology, Jena University Hospital, Jena

Mathias W. Pletz

Institute for Infectious Diseases and Infection Control, Jena University Hospital

Christian Brandt

Institute for Infectious Diseases and Infection Control, Jena University Hospital

\section{Case Report}

Keywords: Nanopore sequencing, Streptobacillosis, Streptobacillus moniliformis, WGS, Zoonosis

Posted Date: February 23rd, 2022

DOI: https://doi.org/10.21203/rs.3.rs-1374822/v1

License: (9) This work is licensed under a Creative Commons Attribution 4.0 International License.

Read Full License 


\section{Abstract}

\section{Background}

Streptobacillus moniliformis is the main cause of human streptobacillosis, also known as rat-bite or Haverhill fever. Generally transferred by bites or scratches of rats and other rodents or by rat-excrement polluted water, symptoms commonly start to appear after $<10$ days and are highly variable. Due to scarcity, non-specific symptoms, and difficulties in cultivating this fastidious organism, the correct diagnosis is frequently delayed.

Case

Here, we report an 85-year old male with a left-sided prosthetic hip joint hospitalized because of immobilizing left hip pain. The patient denied fever, chills, and night sweats. There was no swelling or erythema. Via sonography an echo-free round mass was detected at the left hip. Punctuation of the hip joint revealed putrid secretion. From the synovial fluid and from concomitantly drawn blood cultures grew a pleomorphic, gram-negative rod after $48 \mathrm{~h}$ and $35 \mathrm{~h}$, respectively. The microorganism formed small, grey, round, and convex colonies. VITEK and VITEK MS (MALDI-TOF) analysis brought no results. Therefore the unknown isolate was sent to the Institute for Infectious Diseases and Infection Control for sequencing. Following isolation, the DNA was sequenced using Nanopore Third-Generation Sequencing. After one hour enough data were generated to identify the microorganism as Streptobacillus moniliformis.

Conclusion

To date, this is the second published case of a periprosthetic bone infection caused by Streptobacillus moniliformis. Although streptobacillosis has a mortality rate of around $10 \%$ in humans, it is rarely diagnosed and published. In this context, nanopore sequencing enables fast and reliable diagnostic results for identifying microorganisms and their properties.

\section{Background}

Streptobacillus moniliformis is the main cause of human streptobacillosis. Depending on the transmission, it is distinguished into "rat-bite"-fever (RBF) and "Haverhill"-fever (HF)[1]. RBF is transferred by bites or scratches of rats and other rodents, whereas HF-transmission occurs by rat-excrement polluted water and food. Symptoms are relatively unspecific, comprising more general symptoms like fever, vomiting, malaise, muscle pain and rash as well as more severe symptoms like arthritis, endocarditis, and bacteremia[2]. They typically start to appear after $<10$ days[3]. Cultivation of Streptobacillus moniliformis proves to be challenging, growing under microaerophilic conditions after 2-7 days[3,4]. Overgrowth by faster-growing bacteria in the specimen can therefore hinder the initial cultivation[4]. Due to the unspecific first symptoms and difficulties in the cultivation, wrong or delayed diagnosis is not unlikely[3]. 


\section{Case Report}

An 85-year-old man was hospitalized with immobilizing pain in the area of his left hip joint prosthesis. He denied typical infection symptoms (e.g., shortness of breath, headache, neck stiffness, nausea, vomiting) when asked. After sonography revealed an echo-free, round mass on the left hip, the patient was diagnosed with a periprosthetic infection of the left hip joint. The hip joint was punctured, and a putrid secretion was isolated and sent to the microbiology department for further analysis.

The laboratory could detect an unknown microorganism after 35 hours of incubation in aerobic blood culture and after 48 hours in the puncture secretion. The colony morphology was described as small, round, convex gray colonies. Blood culture microscopy showed the microorganism as flagella-like, gramnegative rods (Figure 1A). In contrast, microscopy of the culture specimen showed gram-negative, pleomorphic rods, partially swollen on their sides (Figure 1B). The unknown microorganism was sensitive to penicillin, amoxicillin / clavulanic acid, ceftriaxone, and tigecycline. VITEK and VITEK MS (MALDI-TOF) were used for bacterial typing, but neither system could determine the species. Therefore, the microbiology department transferred the sample material to the Institute for Infectious Diseases and Infection Control for rapid whole-genome sequencing (WGS).

The isolate could be reliably classified as Streptobacillus moniliformis 7.5 hours after the arrival of the material (DNA isolation 2.5 hours, sequencing library four hours, sequencing one hour). To speed up the bacterial identification, we utilized that the sequencing data of the nanopore sequencer are available at any time, even while the sample is still being sequenced. Therefore, after approximately one hour of sequencing, 10,000 raw DNA fragments (reads) were classified directly with Sourmash[5] using the GTDB database (Release 06-RS202 (April 27, 2021))[6, 7]. We transferred the preliminary results directly to the microbiology department, and sequencing continued overnight. The subsequent complete reconstruction of the entire genome generated a circular 1.65 megabase large genome, which confirmed the preliminary results (genome is available under the accession number: CP079714). In agreement with the phenotypic antibiotic resistance testings, no resistance genes could be identified on the genome (genotype).

\section{Discussion}

Streptobacillosis is rarely diagnosed[8, 9], even though $10-100 \%$ of domestic and $50-100 \%$ of wild rats are infested with Streptobacillus moniliformis[3]. The risk of infection by bite is assumed to be around $10 \%$ [3], but other rodents or excrement-polluted food or water can also cause the infection[1]. If left untreated, the disease has a mortality rate of approximately $10 \%[3]$.

Periprosthetic bone infection caused by Streptobacillus moniliformis was first reported in 2020 by Smallbones et al., describing a case of infection in a knee replacement[10]. Therefore this publication is the second reported case with a periprosthetic focus in the worldwide literature.

We conducted literature research of published case reports via PubMed and found 256 Publications using "Streptobacillus moniliformis" as a search term (August 2021), excluding 139 non-case reports. We 
further excluded 18 publications as they lacked information or were not eligible for inclusion as a Streptobacillus moniliformis infection (see Supplementary Data for details).

The remaining 99 publications contained information on the number of reported cases and the focus of the infection. All cases were confirmed by culture or PCR. Survival, gender, age, and country of origin were extracted from the references. Time of survival after infection was not consistently reported in the case histories. Hence, the following case fatality rates are not based on uniform periods but reflect a summarized estimation. $36.9 \%$ were female ( 41 out of 111 ). The median age was 36 . The overall casefatality rate was $9.9 \%$ (11 out of 111 cases; 93 not fatal; 7 unknown). Most cases were reported from the USA (33.3\%; 37 out of 111) followed by the UK (11.7\%; 13/111), Japan (7.2\%; 8/111), and France, Germany, and Spain (4.5\% each; 5/119). 57 publications with 64 cases $(57.7 \% ; 64 / 111)$ referred to bloodstream infections with positive blood cultures. The mortality of bloodstream infection was $12.5 \%$ (8/64). Fifteen publications with 17 cases $(15.3 \% ; 17 / 111)$ reported endocarditis as the focus of infection. The case fatality rate was $35.2 \%(6 / 17)$. In $12(18.8 \% ; 12 / 64)$ cases with bloodstream infection, endocarditis was proven simultaneously. Those cases showed a mortality of $41.6 \%(5 / 12)$. In 40 cases $(36.0 \% ; 40 / 111)$ arthritis was reported (mortality $2.5 \% ; 1 / 40)$. Other foci as meningitis, CNS Infection, Osteomyelitis, Spondylodiscitis, Chorioamnionitis, epidural abscess, and abscesses of spleen, brain, skin, psoas, or the small pelvis were rare.

Slow growth and specific growth conditions can be significant obstacles in the identification of Streptobacillus moniliformis. The slower metabolism may cause weaker fluorescent biochemical reactions in the VITEK system that hampers reliable species identification in general[11-13]. However, even the VITEK MS system might fail to provide an accurate, species-level identification ranging from 1.5 to $22.2 \%$ in gram-negative Isolates depending on the reporting study $[14,15]$. The failure to identify a species can have multiple origins, like unspecific or conflicting mass spectra, or due to the rarity of the disease, not enough good database references.

The most precise form of species identification remains complete genome sequencing for accurate typing and genotyping (e.g., resistance or virulence genes)[16]. Highly curated genome databases such as GTDB with over 250,000 different bacteria and archaea can reliably identify even close relatives of unknown bacteria. In addition, long-read sequencing technologies such as PacBio or Nanopore sequencing already generate information-rich raw data (based on the length of the DNA) to enable reliable species identification without genome reconstruction. This approach was used to identify the Streptobacillus moniliformis at the beginning of the sequencing run, reducing the time to results by half a day. The current barriers to broader routine-based sequencing applications remain price per sample and the bioinformatic know-how required to analyse sequencing data. However, prices are getting cheaper, and raw data is getting more accurate. In addition, scientists have been working very actively in recent years on so-called workflows to simplify the downstream analysis of sequencing data and reduce the bioinformatics bottleneck in applied science[17-19].

\section{Conclusion}


Streptobacillus moniliformis is a slow-growing pathogen that confers the rarely diagnosed zoonotic disease streptobacillosis. The slow-growing nature of this organism makes it especially difficult to identify via VITEK and the VITEK-MS, the current standards in routine clinical microbiology. Utilizing internal sequencing solutions designed for rapid typing can provide reliable results for all known bacteria or archaea species within a day. Further, WGS-analysis represents a "one-fits-all" approach as sequencing preparation between microorganisms remains mostly the same while also providing genotyping besides species identification.

Sequencing is slowly becoming an essential tool for the routine microbiology laboratory besides outbreak tracing. The price per sample is continuously getting cheaper, new miniature device options are available, and bioinformatic workflows that simplify the downstream analysis are broadly available to nonscientific personnel.

\section{Abbreviations}

CNS - central nervous system

HF - "Haverhill"-fever

RBF - "rat-bite"-fever

MS - mass spectroscopy

WGS - whole-genome sequencing

\section{Declarations}

\section{Acknowledgements}

Not applicable

\section{Author contributions}

Sample collection, microbiological testing and imaging, B.E.; sequencing, R.S.; software, C.B.; bioinformatic analysis, R.S., C.B.; literature research, B.S.; writing first draft, R.S.; reviewing and editing manuscript, R.S., C.B., B.S. and M.W.P.; supervision, C.B.; project administration, C.B.; funding acquisition, M.W.P. All authors have read and agreed to the published version of the manuscript.

\section{Availability of data and materials}

Not applicable 


\section{Competing interests}

The authors have declared that no competing interests exist.

\section{Consent for publication}

The patient gave his written agreement to the publication of this work.

\section{Ethics approval and consent to participate}

Not applicable

\section{Funding}

This work was supported by grants from the Federal Ministry of Education and Research, [grant number 13GW0423B].

\section{References}

1. Gaastra W, Boot R, Ho HTK, Lipman LJA. Rat bite fever. Vet Microbiol. 2009;133:211-28.

2. Eisenberg T, Ewers C, Rau J, Akimkin V, Nicklas W. Approved and novel strategies in diagnostics of rat bite fever and other Streptobacillus infections in humans and animals. Virulence. 2016;7:630-48.

3. Elliott SP. Rat bite fever and Streptobacillus moniliformis. Clin Microbiol Rev. 2007;20:13-22.

4. Eisenberg T, Ewers $C$, Rau J, Akimkin V, Nicklas W. Approved and novel strategies in diagnostics of rat bite fever and other Streptobacillus infections in humans and animals. Virulence. 2016;7:630-48.

5. Brown CT, Irber L. sourmash: a library for MinHash sketching of DNA. J Open Source Softw. 2016;1:27.

6. Parks DH, Chuvochina M, Waite DW, Rinke C, Skarshewski A, Chaumeil P-A, et al. A standardized bacterial taxonomy based on genome phylogeny substantially revises the tree of life. Nat Biotechnol. 2018;36:996-1004.

7. Chaumeil P-A, Mussig AJ, Hugenholtz P, Parks DH. GTDB-Tk: a toolkit to classify genomes with the Genome Taxonomy Database. Hancock J, editor. Bioinformatics. 2019;btz848.

8. Matt U, Schmiedel J, Fawzy A, Trauth J, Schmidt K, Vogel K, et al. Infection in a Young Immunocompetent Male Caused by Streptobacillus felis, a Putative Zoonotic Microorganism Transmitted by Cats. Clin Infect Dis. 2021;72:1826-9.

9. Hagelskjaer L, Sørensen I, Randers E. Streptobacillus moniliformis infection: 2 cases and a literature review. Scand J Infect Dis. 1998;30:309-11. 
10. Smallbones M, Monem M, Baganeanu M, Okocha M, Sofat R. Near-fatal Periprosthetic Infection with Streptobacillus moniliformis: Case and Review. J Bone Jt Infect. 2020;5:50-3.

11. Automated antimicrobial susceptibility testing of slow-growing Pseudomonas aeruginosa strains in the presence of tetrazolium salt WST-1 - ScienceDirect [Internet]. [cited 2021 Jul 28]. Available from: https://www.sciencedirect.com/science/article/pii/S0167701221001202

12. Ling TKW, Liu ZK, Cheng AFB. Evaluation of the VITEK 2 System for Rapid Direct Identification and Susceptibility Testing of Gram-Negative Bacilli from Positive Blood Cultures. J Clin Microbiol. 2003;41:4705-7.

13. Eisenberg T, Nicklas W, Mauder N, Rau J, Contzen M, Semmler T, et al. Phenotypic and Genotypic Characteristics of Members of the Genus Streptobacillus. Janssen PJ, editor. PLOS ONE. 2015;10:e0134312.

14. Yoo IY, Han J, Ha SI, Cha YJ, Pil SD, Park Y-J. Clinical performance of ASTA SepsiPrep kit in direct bacterial identification and antimicrobial susceptibility test using MicroIDSys Elite and VITEK-2 system. J Clin Lab Anal. 2021;35:e23744.

15. Manji R, Bythrow M, Branda JA, Burnham C -a. D, Ferraro MJ, Garner OB, et al. Multi-center evaluation of the VITEK® MS system for mass spectrometric identification of non-Enterobacteriaceae Gramnegative bacilli. Eur J Clin Microbiol Infect Dis Off Publ Eur Soc Clin Microbiol. 2014;33:337-46.

16. Viehweger A, Marquet M, Hölzer M, Dietze N, Pletz M, Brandt C. Adaptive nanopore sequencing on miniature flow cell detects extensive antimicrobial resistence [Internet]. Microbiology; 2021 Aug. Available from: http://biorxiv.org/lookup/doi/10.1101/2021.08.29.458107

17. Hodcroft EB, De Maio N, Lanfear R, MacCannell DR, Minh BQ, Schmidt HA, et al. Want to track pandemic variants faster? Fix the bioinformatics bottleneck. Nature. 2021;591:30-3.

18. Ewels PA, Peltzer A, Fillinger S, Patel H, Alneberg J, Wilm A, et al. The nf-core framework for community-curated bioinformatics pipelines. Nat Biotechnol. 2020;38:276-8.

19. Brandt C, Krautwurst S, Spott R, Lohde M, Jundzill M, Marquet M, et al. poreCov-An Easy to Use, Fast, and Robust Workflow for SARS-CoV-2 Genome Reconstruction via Nanopore Sequencing. Front Genet. 2021;12:711437.

\section{Figures}




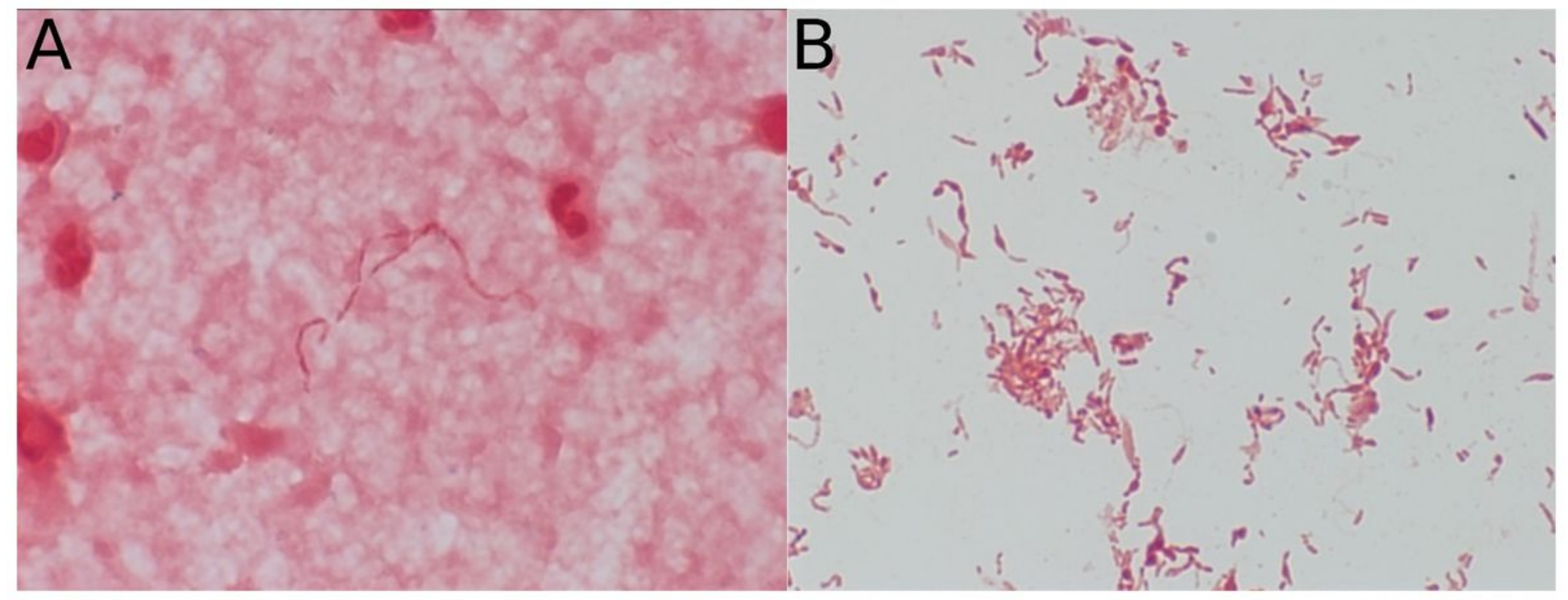

\section{Figure 1}

A) Flagella-like, gram-negative Streptobacillus moniliformis-rods in blood culture. B) Gram-negative, pleomorphic Streptobacillus moniliformis-rods in culture of the hip joint-secretion.

\section{Supplementary Files}

This is a list of supplementary files associated with this preprint. Click to download.

- 2021SpottcasereportSmoniliformissuppl.docx 